\title{
Panorama de la Sociedad de la Informacion en América Latina
}

\author{
Fortunato Contreras Contreras \\ Departamento Académico de Bibliotecologia y \\ Ciencias de la Información
}

\section{Iniroducción}

En las últimas décadas se está viviendo lo que en la década del 60 algunos autores denominaban sociedad del conocimiento o sociedad de la información. Esto ocurrió debido a la evolución de la sociedad industrial, que producia bienes de consumo, a una sociedad dominada por la información en todas las esferas de la vida humana.

La era industrial se caracterizaba por el uso del capital, el dinero y recursos tangibles. En el presente son la información y el conocimiento los recursos básicos y fundamentales.

En pleno siglo XXI épocat de cambios rápidos, impredecibles, la única cosa con que podemos contares lacerteza de que el cambio remueven la base del mundo que nos rodea. Cualquier cosa que hagamos y dondequiera que la hagamos (estilos de trabajo, condiciones económicas, tecnologias, organizaciones, comunicaciones, estilos de vida, etc.) cambia vertiginosamente. Las técnicas, los conocimientos y los productos de hoy viven rápidamente, se envejecen antes de tiempo y mueren jóvenes. Se pide aprender, hacer, producir más con menos recursos y sin perder tiempo. Estamos asistiendo sin duda alguna a un nuevo modelo de sociedad en el que la información, entendida como conocimiento acumulado de forma comunicable, aparece como el cimiento del desarrollo económico, político y social. Esta concepción de la sociedad basada en la información y el conocimiento impone progresivamente, en todos los palses, el establecimiento de políticas de información, llevando a sus gobiemos a comprometerse en acciones que van desde los diagnósticos y estudios, hasta la creación de un aparato estatal, más o menos desarrollado, que permita controlar y promover las actividades de información cientifica y técnica. 
A través de la historia del hombre, la información ha sido una premisa para el desarrollo social e individual. En la inminente Sociedad de la Información estos planteamientos son aún más evidentes: las sociedades desarrolladas y ricas en información tienen acceso a una tecnologia avanzada, mientras que las sociedades en vías de desarrollo dependen de aquéllas para su desarrollo industrial y/ o cultural. Por ello imitan sus estructuras y recogen sus planteamientos en materia de politicas de información. Este problema tendrá solución si, por un lado, los gobiernos establecen politicas definitivas para el desarrollo de Sistemas Nacionales de Información que asistan a la investigación cientifica y técnica, y por otro, si los distintos paises se coordinan en programas de cooperación a fin de ahorrar recursos y elevar su potencial en materia de información, estableciéndose Politicas Internacionales de Información.

\section{Sociedad de la información}

A. Cornella (1999) define a la sociedad de información "Como una sociedad en que la información se usa intensivamente en la vida social, cultural, económica y politica".

Para I. Nuñes Paula (1999):

La sociedad de la información no es algo del futuro sino del presente, la sociedad ha sido definida como una sociedad en la que el desarrollo de los servicios, de la educación, de la cultura y de todas las esferas de las actividades de la vida humana, dependerán de la información que se tenga y una sociedad que necesita que la mitad de la fuerza laboral este dedicada directamente a la gestión de la información. Esa esfa socfedad de hoy y aún mas" es la sociedad de siempre ...

Para Isidro Fernández-Aballi (1999) "La Sociedad de la Información es el ambiente social que resulta de la apropiación y utilización de la información a gran escala".

Para Alvaro Agudo (2001):

La Sociedad de la Información es un hecho permanentemente reconstruido por actores que pertenecen a sectores sociales de la gran mayoria de los paises del globo. Actores que interactúan supra y transnacionalmente por medio del conjunto integrado, a través de Internet, de infraestructuras nacionales de información para ofrecer, vender, consumir y utilizar información y conocimiento.

Dada la multiplicidad de conceptos, definiciones, significados que se atribuyen a la Sociedad de la Información y ya que no se ha impuesto una definición que pueda reconocer como aceptada comúnmente, se considera conveniente 
concretar el significado que se da a la expresión sociedad de la información aceptada por la mayoria de autores:

Sociedad de la Información es un estadio de desarrollo social caracterizado por la capacidad de sus miembros (ciudadanos, organizaciones, Estado) para acceder, obtener y compartir información instantáneamente desde cualquier lugar y en la forma adecuada, fundamentalmente mediante el uso de la Tecnología. Como vemos en la figura 1 , la sociedad de la información ha tenido la siguiente evolución.

Fig. 1. Evolución de la Sociedad Moderna

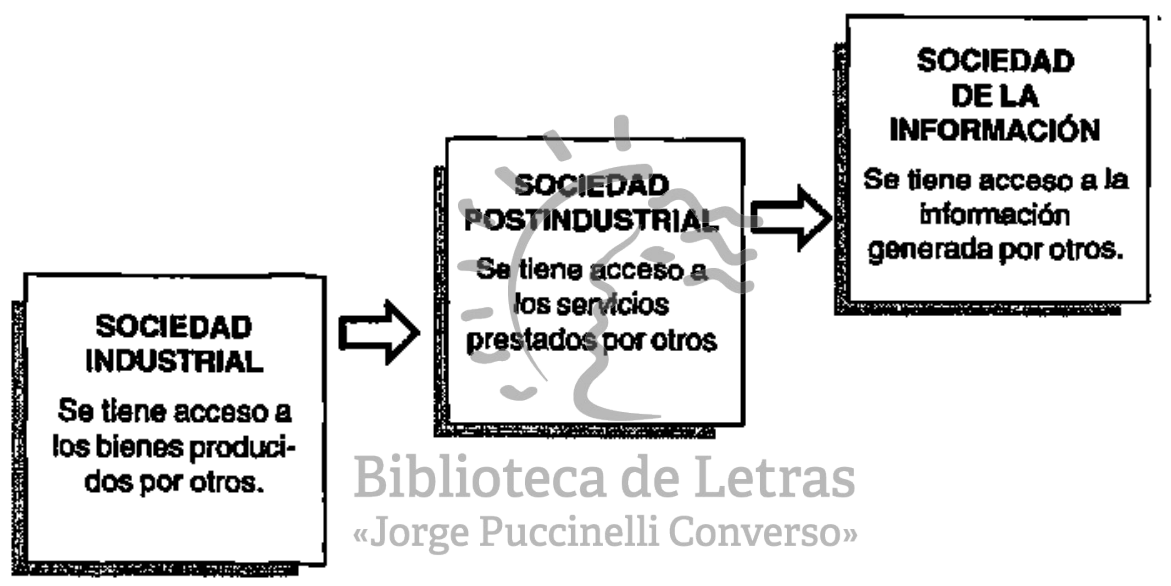

Es necesario aclarar que este concepto se aplica con toda la extensión de la palabra en los paises desarrollados o industrializados y con menor o relativa aplicación en países subdesarrollados.

Existe hoy un reconocimiento, ya tácito, de que la información constituye un recurso nacional indispensable para todos los programas y actividades nacionales de desarrollo.

Las nuevas condiciones en que se desarrolla el mundo, caracterizado por una serie de cambios acelerados, condicionan un mayor uso de la información en función de la toma de decisiones operativas, tácticas y estratégicas, y en la solución de los múltiples problemas que plantea la aspiración de crear las bases para un desarrollo sostenible. 
Por ello, la información es considerada, por muchos, como un recurso estratégico, comparable con la energia, el trabajo y el capital y se conceptualiza, cada vez más, como símbolo de poder.

El inminente desarrollo de una nueva Sociedad o una nueva Era, basada en el uso intensivo de la información y el conocimiento se abre paso y, en consecuencia, la forma de producir, educarse y recrearse de la Sociedad se está revolucionando; tanto es el peso que ello va adquiriendo, que actualmente se apunta la necesidad de considerar un sector cuaternario en la economía: El de la Información.

Para que ello pueda hacerse realidad, es preciso desarrollar lo que se ha dado en llamar la infraestructura de la información, compuesta por: el factor humano, los contenidos de información, el equipamiento y la infraestructura material (desde los medios informáticos hasta las telecomunicaciones), el software y los mecanismos de intercambio electrónico de información; todo ello unido a elementos regulatorios y de política y a los recursos financieros necesarios para tales desarrollos.

Los indicadores utilizados para medir el desarrollo de la información de los países están relacionados a:

- La mayor cantidad de trabajadores de la información

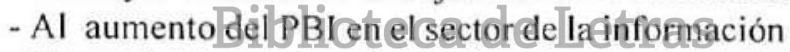

- El uso de nueyas tecnolegiaselli Converso"

En el caso del uso de la tecnología, el Banco Mundial (Cuadro $\mathrm{N}^{\circ} 1$ ), usa como indicadores de medición de la sociedad de la información:

- Aparatos de radio

- Número de televisores

- Usuarios de cable

- Teléfonos móviles

- Líneas telefónicas

- Faxes

- Computadoras personales

- Usuarios de Internet. 


\section{Cuadro $\mathrm{N}^{\circ} 1$}

Sociedad de la Información

\begin{tabular}{|c|c|c|c|c|c|c|c|c|c|}
\hline \multirow[t]{2}{*}{ País } & 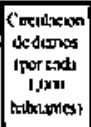 & 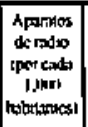 & 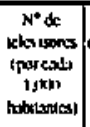 & 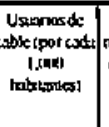 & 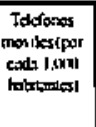 & 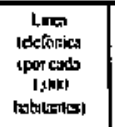 & 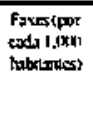 & 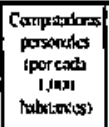 & 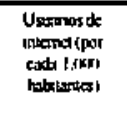 \\
\hline & 1996 & 1997 & 1998 & 1998 & 1998 & 2000 & 1998 & 1998 & 2000 \\
\hline Alcmania & 311 & 948 & 580 & 21450 & 170 & 60,12 & 73.1 & 304.7 & 2920.57 \\
\hline Argentina & 123 & 681 & 289 & $163, \mathrm{I}$ & 78 & 21.32 & 2,0 & 44.3 & 675,9 \\
\hline Nustralia & 293 & 1376 & 639 & 43,6 & 286 & 52,41 & 48,6 & 411.6 & 3497,42 \\
\hline Austria & 296 & 753 & 516 & 139.1 & 282 & 47,36 & - & 233.4 & $2,551,54$ \\
\hline Bélgica & 160 & 793 & 510 & 367.3 & 173 & 69.94 & 18,7 & 286,0 & $1,968.31$ \\
\hline Brasil & 40 & 444 & 316 & 16.3 & 47 & 18,18 & 3,1 & 30,1 & 293,92 \\
\hline Canadá & 159 & 1077 & 715 & 263,8 & 176 & 67,63 & 33,3 & 330,0 & $4,130.07$ \\
\hline Chile & 98 & 354 & 232 & 44,8 & 65 & 22.52 & 2,7 & 48,2 & $1,155,31$ \\
\hline China & - & 333 & 272 & 40,0 & 19 & 11,2 & 1,6 & 8.9 & 173,70 \\
\hline Colombia & 46 & 581 & 217 & 16,7 & 49 & 16,92 & 4.8 & 21,9 & 207,46 \\
\hline Corca del Sur & 196 & 147 & 53 & & & 46,37 & - & - & $4,025,37$ \\
\hline Cuba & 118 & 353 & 239 & $>$ & - & 4,36 & - & - & 53,38 \\
\hline Dinamarca & 309 & 1141 & 585 & 248.4 & 364 & 75,25 & - & 377,4 & $3,658,52$ \\
\hline España & 100 & 333 & 506 & 11,8 & 179 & 42,12 & 17,8 & 144,8 & $1,327,04$ \\
\hline Estados Unidos & 215 & 2146 & 847 & 244,3 & 256 & 69.97 & 78,4 & 458,6 & $3,468,78$ \\
\hline Finlandia & 455 & 1496 & 640 & 175,7 & 572 & 54,69 & 38,5 & 349,2 & $3,722,95$ \\
\hline Francia & 218 & 937 & $60 I$ & 27,5 & 188 & 58.02 & 47.4 & 207.8 & $1,445,58$ \\
\hline Grecia & 153 & 477 & 466 & 1,2 & 194 & 53.16 & 3,8 & 51.9 & $939,4 !$ \\
\hline Guatemala & 33 & 79 & 126 & 28.5 & 10 & 5,75 & - & 8.3 & 58,61 \\
\hline Holanda & 306 & 978 & 5430 & 378,3 & $2 B$ & 60.67 & 38,4 & 317,6 & $2,381,26$ \\
\hline India & - & 121 & 69 & 18,8 & elli & 3,20 & 0,2 & 2,7 & 49,39 \\
\hline Irlanda & 150 & 699 & 403 & 171.1 & 257 & 42,63 & 27,4 & 201,7 & $2,101,89$ \\
\hline Israel & 290 & 530 & 318 & 184.0 & 359 & 46.25 & 24.9 & 207.2 & $1,764,50$ \\
\hline Italia & 104 & 878 & 486 & 2,8 & 385 & 47,39 & 31,3 & 173.4 & $1,047.16$ \\
\hline Japón & 578 & 955 & 707 & 114,8 & 374 & 65.33 & 126,8 & 232.2 & $3,709,45$ \\
\hline México & 97 & 325 & 261 & 15,7 & 35 & 12,47 & 3,0 & 47,0 & 274,31 \\
\hline Perú & 84 & 273 & 144 & 14,1 & 30 & 6,37 & (n) & 18,1 & 158,64 \\
\hline Polonia & 113 & 523 & 413 & 83,3 & 50 & 28,24 & - & 43.9 & 722,30 \\
\hline Portugal & 75 & 304 & 542 & 59,8 & 309 & 43,06 & 7,0 & 81.3 & $5,950,10$ \\
\hline Reino Unido & 329 & 1436 & 645 & 45,9 & 256 & 56.72 & 33,9 & 263,0 & $2,576.72$ \\
\hline República Checa & 254 & 803 & 447 & 71,1 & 94 & 32,79 & 10,4 & 97,3 & 976.18 \\
\hline Rusia & 105 & 418 & 420 & 38.3 & 5 & 21,50 & 0,4 & 40,6 & 210,98 \\
\hline Singapur & 360 & 822 & 348 & 49,5 & 346 & 48,45 & 31,6 & 458,4 & $2,986,78$ \\
\hline Suecia & 445 & 932 & 531 & 221,4 & 464 & 68.30 & 50,9 & 361.4 & $4,558,29$ \\
\hline Suiza & 337 & 1000 & 535 & 352.7 & 235 & 71.99 & 29,2 & 421.8 & $2,978,62$ \\
\hline Turquia & 111 & 180 & 286 & 9.2 & 53 & 28,00 & 1,7 & 23,2 & 304,41 \\
\hline
\end{tabular}

FUENTE: BANCO MUNDIAL. World development indicasors 2000-2001. 
Como pueden apreciar, las unidades de información no constituyen indicadores de desarrollo de la sociedad de la información, porque se sobreentiende que esta etapa ya ha sido superada, vale decir que, en todos los países existen o debería existir un desarrollo de alto nivel de las unidades de información y de los sistemas nacionales de información. Esto se puede decir que solamente ocurre con los paises desarrollados, mas no en los paises subdesarrollados en especial en América Latina.

\section{Evolucion histórica de la expresión sociedad de la información}

Su origen se remonta a bastante años atrás, cuando algunos especialistas percibieron los primeros indicios de que la Sociedad Industrial comenzaba a transformarse en un tipo de sociedad distinta, en la que el control y optimización de los procesos industriales empezaba a ser sustituido en cuanto a clave económico por el control y manejo de la información.

Progresivamente se fue percibienđo que la emergente sociedad de la información se iba caracterizando por la posición central y relevante de la información y de las tecnologias asociadas a la vez como factores de producción y como productos. Esta evolución y formación del concepto se resumen en las siguientes épocas:

En 1960: El surgimientodellconcepte de Sociedad de la Información, puede ser ubicado en la década del sesenta y cornienzos de los setenta, principalmente por Alain Touraine y su contraparte estadounidense Daniel Bell. Ambos analizaron los cambios sociales y económicos en la sociedad de ese tiempo y utilizaron la categoría de post-industrialismo, para indicar que una nueva era estaba aproximándose. En su análisis, Touraine se enfocó en los cambios en las relaciones de poder entre las clases sociales, y en la aparición de una nueva clase social de tecnócratas, la cual consideró que llegaría a ser dominante.

En 1962 Fritz Machlup: "Producción del conocimiento". Machlup de la Universidad de Princeton, intentó describir el significado económico del desarrollo de las actividades de información y comunicación proporcionando un ejemplo de cálculo de valor monetario de este tipo de producción que se denomina producción de conocimiento. 
En 1969 MITI de Japón: "Hacia la Sociedad de la información Plan JACUDI". El Ministerio de Industria y Comercio Japonés publica un informe del Industries Structure Council titulado Toward the information society, sobre la tesis de este informe en 1972 una organización no lucrativa japonesa el Japan Computer Usage Development Institute (JACUDE), presentó al gobierno el Plan para la Sociedad de la Información: un objetivo nacional para el afio 2000 conocido universalmente como Plan JACUDI y que fue el primer apunte estratégico sobre las políticas públicas en la sociedad de la información.

1974 Marc Porat: "Economía de la Información". Marc Porat de la Universidad de Stanford, publica un trabajo en el que sostiene que las actividades relacionadas directa o indirectamente con la información se singularizan progresivamente. Delínea así un nuevo campo de la actividad productiva, el de la economía de la información, unido siempre al desarrollo de las nuevas tecnologías y que adquiere una personalidad diferenciada y autónoma dentro del sector terciario.

1978 Nora-Minc: "La telemática". Simon Nora y Alan Minc publican un informe titulado L'informatisation de la Sociéte. La atención en este caso, se presta al desarrollo de la industria o del sector de los servicios informáticos y de telecomunicaciones. Se presenta por primera vez el neologismo telemática.

1978 John Naishbitt: "Megatendencias". John Naishbitt publica Megatrends obra que comenzó a ser conocida yutilizada la expresion Sociedad de la Información y donde se describian los escenarios futuros a los que daría lugar la Sociedad de la Información

1980 Yoneji Masuda: "La sociedad de la información". Yoneji Masuda, fundador y presidente del Instituto para la Sociedad de la Información y profesor de la Universidad Japonesa de Aumori, fue director de JACUDI. Masuda publicó en 1980 su libro The information society as a post-industrial society, que se tradujo al espafiol como La sociedad informatizada como sociedad postindustrial. Obra que tuvo gran difusión y no solamente popularizó la expresión sociedad de la información sino que su estudio prospectivo de la sociedad de la información ha contribuido a marcar las pautas de muchos planes estratégicos actualmente vigentes.

El concepto de la sociedad de la información y el escenario futurista de la política japonesa fueron adoptados con mucho entusiasmo por visionarios occi- 
dentales como Alvin Tofler, John Naisbitt y más recientemente Nicholas Negroponte. Todos contribuyeron a la popularización de las ideas de Touraine y Bell y a la diseminación del concepto de sociedad de la información y sus sinónimos, como la sociedad digital, la sociedad interconectada (posteriormente transformada a la sociedad inalámbrica), la cabaña electrónica, la aldea global y otros similares.

Una visión sobre la sociedad de la información en los paises desarrollados

La Comisión Europea ha hecho uso de la expresión sociedad de la información en múltiples ocasiones y como título de diversas iniciativas, englobando en el concepto de sociedad de la información todos los servicios prestados con el concurso de las tecnologías de la información y las comunicaciones (TIC)

La Conferencia Ministerial de 1995 de la OCDE requirió al Comité de Políticas de Información, Informática y Comunicaciones para que desarrollara recomendaciones para la explotación de las oportunidades de las infraestructuras Globales de la Información-Sociedad de la Información

La Organización de las Naciones Unidas para la Educación, la Ciencia y la Cultura (UNESCO), ha establecido un Observatorio sobre la Sociedad de la Información, con el fin de tratary difunder losaspeetos éticos, legales y sociales de ésta, en constantęeyoluciónccinelli Converso»

Un importante número de países ha puesto en marcha en los últimos años iniciativas para el desarrollo o simplemente la promoción de la sociedad de la información. En sus títulos suele introducirse la expresión sociedad de la información. Son los casos de Reino Unido (Information Society Iniciative), Francia (Programme dactioon gonvernemental pour la société de l'information), Finlandia (Finlands Way to the information society), España (Info XXI, la sociedad de la información para todos).

En un reciente discurso, el Sr. Ngubane, Ministro de Arte, Cultura, Ciencia y Tecnología de Sudáfrica, pronunciado durante la " 6 a Olimpiada Internacional de la Mente" (París, 16-17 de noviembre de 2000)1, cita al economista Lester Thurow diciendo que "En el léxico de Thurow la segunda revolución industrial fue la era de la información y ahora, por primera vez en la historia, la gran 
riqueza dependerá cada vez más del control del conocimiento, no del control de los recursos naturales tales como la tierra, el oro y el petróleo". En el mismo artículo, el Ministro Ngubane utiliza el Informe Mundial sobre el Desarrollo Humano del PNUD 1999 para advertir sobre las "desigualdades globales", que se han incrementado con la globalización a una tasa acelerada tanto dentro como entre los países. También se hace referencia a las desigualdades de acceso a la Intemet y su implicación en el acceso a la información de todo tipo.

Si deseamos discutir la planificación de políticas hacia la sociedad de la información, el documento "Green Paper on the Information Society in Portugal" es pertinente para académicos, políticos y formuladores de política. Este trata once áreas principales, a saber:

I. Carácter Democrático de la Sociedad de la Información.

2. Gobiermo Abierto.

3. Conocimiento Disponible

4. Escuelas Inter-conectadas: Aprendiendo en la Sociedad de la Información.

5. La Empresa Comercial en la Sociedad de la Información.

6. El Empleo en la Sociedad de la Información.

7. El Mercado y la Sociedad de la Información.

8. Implicaciones Sociales de la Sociedad de la Información.

9. Implicaciones Legales de la Sociedad de la Información.

10. La Infraestructura Nacional de Información.

11. Investigación Yy Desarrollo en la Sociedad dela Información.

Mas recientemente, en su artículo "Gobernando la Sociedad del Conocimiento Global", Philippe Quéau, Director de la División de la Sociedad de la Información de la UNESCO, subraya que:

No se puede comparar al conocimiento con otros productos. El conocimiento posee propiedades muy especificas que difieren sensiblemente de los productos industriales. Tal como el fuego, puede propagarse rápidamente con viento favorable, prácticamente sin costo alguno. La "economfa de red" muestra que el conocimiento puede permitir tanto retomos extremadamente altos como ningún retorno en absoluto. Además, es discutible que el conocimiento no sea realmente el producto sino un modo de pensar. Puede incluso llegar a ser un modo de vida, tal como lo muestran los eruditos. Puede llegar a tener fuertes efectos no lineales, profundos impactos 
sociales y politicos. Por esta razón, la llamada "Brecha Digital" consiste esencialmente en una división social y económica que únicamente se ve agravada por el mero poder de las TI.

Los organismos internacionales también han tratado temas relacionados con la Sociedad de la Información, o su versión más idealista, la sociedad del conocimiento. En particular, cabe mencionar las "Recomendaciones sobre Politicas para la Acción 4 en la Infraestructura de la Información Global-Sociedad de la Información Global (GII-GIS)" emitidas en la Reunión del Consejo de la OCDE en 1995.

En dicha reunión, los Ministros de la OCDE consideran que el desarrollo de una economía de la información en red requiere:

- disponibilidad y difusión de infraestructuras interactivas de alta velocidad;

- acceso y uso equitativo de infraestructuras tanto para clientes como para proveedores;

- interconexión e interoperabilidad de infraestructuras y servicios;

- crecimiento y desarrollo de los serviciosmultimedia;

- transacción y salvaguarda de la información, que aseguren la privacidad, la confidencialidad de la información, la seguridad en los pagos y la protección de la propiedad intelectual.

En la UNESCO, i töbsertatoriodela Seciedad de la Información" y el Informe National InformaticsPPlicies andStrategies, proporciona un conjunto muy relevante de información y documentos para personas responsables de planificación de Sociedad de la Información. Para cerrar esta breve introducción y teniendo en cuenta la importancia de desarrollar políticas en los paises, es necesario resaltar dos importantes iniciativas internacionales recientes:

- Grupo de Trabajo de Tecnologías de Información y Comunicación (ICT), cuyo Secretario General emitió un Informe 7 que fue examinado recientemente en la Reunión ECOSOC, los dias 3-4 Mayo 2001 (Agenda item 2)

- La Carta de Okinawa sobre Sociedad de la Información y el Establecimiento de un Grupo de Trabajo de una Oportunidad Digital (DOT)

Ambas iniciativas tratan detalles sobre "Digital Divide" y DOT cubre aspectos más amplios sobre cooperación internacional sobre asuntos de la 
sociedad de la información, de los cuales todos nosotros deberíamos estar conscientes.

Sociedad de la Información en América Latina

Bien distinta es la situación de las economias emergentes de América Latina, sumergidas en una situación económica, política y social, que nada tiene que ver con la panacea descrita. Los gobiernos de estas naciones tienen ante sí problemas cruciales que resolver como el empleo, la educación (bibliotecas), hambre, la insalubridad, el analfabetismo, no el informacional, sino el básico, para una parte importante de su población. Sus infraestructuras de información en consecuencia son débiles y los recursos financieros para el desarrollo de las mismas son prácticamente inexistentes.

El desafio para las economías emergentes parece estar claro; lo primero, que habrá que comprender, es que no se trata de equiparar nuestros palses con los desarollados. Las preocupaciones de los más pobres no pueden estar en el orden de alcanzar los altisimos niveles de vida que, de manera inmediata, se propone el Primer Mundo, a través de estos nuevos desarrollos que se plantean, porque ello, además de irrealizable, desviaría los esfuerzos y recursos por un camino equivocado.

El problema radica en encontrar la vía, a partir de las condiciones actuales que las economlas emergentes presentan, para poné la información en función del desarrollo nacional, como bien ccónómico y como recurso productivo y tratar de lograr una inserción, lo más ventajosa posible, en el contex to internacional, evitando la transculturación que puede traer aparejado este fenómeno y utilizándoto como instrumento para reafirmar y engrandecer su patrimonio y preservar su identidad como naciones.

Nuestros paises definidos como "pobres en información", de "8infopobres", carecen de una adecuada infraestructura informacional, cuyos elementos más significativos son:

- Dispersión de los recursos de información.

- Déficit de Unidades de información y Ausencia de Sistemas Nacionales de información.

- Desarrollo desigual de la infraestructura tecnológica y técnica.

- Disparidad en los criterios de organización de la información lo cual dificulta su acceso y recuperación. 
- Ausencia de meta-información, esto es información sobre la información (fuentes de referencia).

- Débiles estructuras cooperativas de servicios de información.

- Inadecuada racionalización de la inversión y optimización de los recursos disponibles.

- Ausencia de políticas nacionales que casen el desarrollo del espacio Tecnológico e informacional con los objetivos macrosociales de desarrollo.

Es precisamente este último aspecto una de las principales debilidades que confrontan los países del área, en tanto, de lograrse la formulación y aplicación de una Política Nacional de Información, que satisfaga los requisitos descritos, contarian con el instrumento (estrategias y lineas de acción) que ofrecería la posibilidad de desarrollar la infraestructura de la información y poner ésta al servicio del desarrollo nacional. Entre las debilidades, que hasta el momento han confrontado los paises de la región en la formulación, aplicación, seguimiento y evaluación de su Política Nacional de Información, se sitúan:

- Limitaciones de índole conceptual relacionadas con todo lo concerniente a la información, agudizadas a la luz de los nuevos desarrollos.

- Falta de claridad en cuanto a qué debe entenderse por Política, cómo concebir el proceso de formulación y legitimización de una decisión y un resultado de política y de cómo introducir de manera efectiva una decisión de esta índole en la agenda gubernamental

- Carencia de unenfoque de esta problemática, partiendo de la interpretación del significadg de los nuevbs desarvellosy las particularidades de su aplicación, de la consideración de su contexto político concreto y de las condiciones específicas del desarrollo nacional.

- Dificultades en la mayoria de los países de la región, en el desarrollo de acciones nacionales efectivas relacionadas con la definición e implementación de estas Políticas.

- Insuficiente estructuración y proyección del sector de la información en los países del área, que ha impedido influir eficazmente en los niveles decisorios (de gobierno y parlamentario) para que una iniciativa de política en este campo, se asocie con una demanda social de cuya satisfacción se derivaría una ganancia política.

- Débil iniciativa en el seno del Gobierno, que sustente un apoyo constante y definido y le dé fuerza política para una efectiva respuesta institucional

- Bajo perfil de la fuerza profesional vinculada al sector de la información, 
básicamente dado por las limitaciones propias de los profesionales de la información, que no han sido formados como agentes sociales para el cambio y que impide que sea identificada, con suficiente claridad, la trascendencia de su rol en el desarrollo económico y social del pais y de la información misma en este proceso.

Unido a las dificultades descritas, se da el caso, de que no existe una clara conciencia de la dimensión del problema al que se enfrentan los paises de la región, no sólo desde el punto de vista individual, sino como bloque. No es suficientemente Sistematizado el seguimiento que se le da al desarrollo de este fenómeno en el primer mundo. Es aún incipiente la introducción de esta problemática en las agendas de los foros regionales, de carácter gubernamental, donde se analizan los problemas del desarrollo del área y no existe, en consecuencia, una visión madura y compartida sobre las oportunidades y amenazas del desarrollo de la Sociedad Global de la Información para la región, que ofrezca una posición de consenso en los Foros internacionales donde se discuten estos temas. No se comprende aún, la necesidad de lograr acciones efectivas, que conduzcan a un proceso de integración regional para minimizar las amenazas y aprovechar, al máximo, las oportunidades

De este fenómeno mundial, en tanto, el bloque europeo, por ejemplo, muestra significativos avances en materia de integración alrededor de esta problemática.

No obstante, los esfuerzos por el desarrollo en ta esfera de la información en la región en estos años, ofrecen un panorama mảs alentador del que existía en las décadas de los 70 y los 80 .

- La región en estos momentos dispone de importantes estructuras, sistemas, redes, servicios y productos, que constituyen fuentes y medios importantes de organización y consulta de información, los cuales no exist'lan anteriormente.

- Procesados o no por la región, se cuenta con una serie de datos e información, en muchos casos altamente especializados.

- La información hoy se confirma, en el contexto regional, como un elemento estratégico, tanto para la toma de decisiones en condiciones de incertidumbre y de alta competitjvidad internacional, como de un recurso, llamado a apoyar el crecimiento económico y la evolución de la sociedad. 
- Se realizan esfuerzos crecientes por los países de la región por concretar Políticas Nacionales de Información, atemperadas a las exigencias actuales.

- Existen organismos regionales especializados, como FID/CLA (Comisión Latinoamericana de la Federación Internacional de Información y Documentación) y el Programa Regional para el Fortalecimiento de la Cooperación entre Redes y Sistemas Nacionales de Información para el Desarrollo en América Latina y el Caribe (INFOLAC), que han realizado y realizan sostenidos esfuerzos por potenciar el desarrollo regional en esta esfera, desde una visión actualizada de los problemas a resolver.

Programas de la Sociedad de la Información orientados al uso de tecnologia en América Latina

Recientemente, los gobiernos de América Latina han lanzado procesos o programas de la Sociedad de la Información que tienen por objetivo lograr una visión holística y financiera de este fenómeno.

Chile ha sido el pionero a este respecto a través de su "Iniciativa sobre la Sociedad de la Información" lanzada por la Comisión Presidencial para la Infraestructura Nacional de Información. La iniciativa chilena ha logrado ser adoptada por distintos gobiernos y puedeser con siderrada como un ejemplo de "mejores prácticas" en planificación gubernamental de la Sociedad de la Información.

México cuenta desde hace tiempo con una comisión nacional de informática, un grupo de reflexión para planificar la política nacional acerca de la tecnología de la información. El país implementa el Plan Nacional de Informática a través de distintas instituciones nacionales. INEGI/DNPI actúa como coordinador nacional de políticas de informática y ha jugado un papel activo en la Región, asl como también promovió en 1999 el importante "Simposio Latinoamericano y del Caribe: las Tecnologías de la Información en la Sociedad"

Brasil también ha lanzado recientemente el Libro Verde de la Sociedad de la Información, actualmente en discusión pública. El "Libro Verde" trata varios temas relevantes tales como: Mercado, Trabajo y Oportunidades, Servicios Universales y Capacitación de la Ciudadanía, Educación para la Sociedad de la Información, Contenidos e Identidad Cultural, el Gobierno al Alcance de todos, 
Tecnologias Clave y sus Aplicaciones, Infraestructura Avanzada y Nuevos Servicios.

Jamaica está logrando "la finalización de la primera fase del "Plan Nacional Estratégico para la Tecnología de la Información". Este país se está embarcando en la segunda parte de un Plan que llama a la formulación de planes detaIlados para cada sector.

Uruguay ha lanzado la iniciativa "Uruguay en Red" cubriendo 5 áreas:

1. Alfabetización telemática.

2. Desarrollo de servicios telemáticos para el ciudadano y las empresas.

3. Modernización de la Administración Pública.

4. Promoción de un mercado eficiente de Telecomunicaciones e Internet.

5. Desarrollo de condiciones de competitividad para el Sector Software.

Otros palses han dado curso a iniciativas sectoriales para aprovechamiento de las tecnologfas de la información asl como para evitar déficits e impactos sociales, tal como informado durante la XVIII Conferencla de Autoridades Iberoamericanas de Informática-CAIBJ 13 (Panamá City, 28-29 Septiembre 2000).

En el Perú se ha constituido una Comisión a cargo del Instituto Nacional de Estadística e Informática para que evalúe y plantée propuestas de desarrollo de una sociedad de información.

Estas iniciativas nacionales van en la dirección adecuada y dan gran esperanza de que se origine gradualmente una visión estratégica de la Sociedad de la Información en todos los palses de América Latina y el Caribe.

\section{Propuestas}

Estas propuestas han sido compiladas y recogidas de organizaciones de carácter regional y local que tienen autoridad académica y de investigación como por ejemplo CEPAL/CLADES, INFOLAC, UNESCO, FID, IFFLA, etc., a partir de la identificación y el análisis de la situación latinoamericana para enfrentarlo, es posible identificar la altemativa que tienen las naciones y la región en su conjunto para enfrentar con éxito su desarrollo en las condiciones actuales. Es imprescindible que la información se convierta en un bien económico y en un 
recurso productivo en favor del desarrollo. Esta doble condición de la información en el plano nacional, supone primero, el fomento y desarrollo de las unidades de información y de sistemas nacionales de información, el fomento y desarrollo de soportes de información, elaborar contenidos de información, como resultado del quehacer científico, técnico, económico, cultural y ambiental del pais, que sirvan de materia prima para la producción de bienes y la prestación de servicios de información, en condiciones competitivas, para satisfacer las necesidades de información del mercado interno y que puedan ser exportados al mercado internacional de la información.

Su utilización como recurso productivo significa, incorporar la información producida internamente y la que se considere necesaria, proveniente del exterior, como elemento que potencie todos los procesos económicos y sociales a partir del enriquecimiento cognoscitivo. Ello debe redundar en mejorar la planificación, la toma de decisiones, potenciar la investigación y la innovación, la competitividad de todos los bienes y servicios provenientes de todos los sectores, y por tanto, de la economia en su conjunto, perfeccionar la dirección de la Sociedad y mejorar la calidad de vida de sus miembros.

Sin embargo, la situación en su propio enfrentamiento es altamente compleja y trae aparejados retos prácticamente en todas las esferas del desarrollo de la Sociedad y un necesario reposicionamiento a la hora de enfocar los problemas del desarrollo nacional.

\section{Biblioteca de Letras}

Si se tiene en çenta que el desarrollo informacional, guarda una relación directa con las políticas educativas y culturales y requiere del concurso de todos los ciudadanos y actores sociales, muchos, marginados hoy de la información, el empleo de las tecnologias y el conocimiento, no resulta difícil concluir que los patrones neoliberales no son precisamente los que propician que esta región pueda enfrentar con éxito los retos actuales y mucho menos los futuros.

Para implementar acciones que tengan consecuencias sociales es necesario la:

- La implantación de una infraestructura nacional de información.

- La conectividad a redes mundiales de información.

- La formación masiva de recursos humanos convenientemente calificados.

Una Infraestructura Nacional de Información debe estar compuesta por:

- Estructura física de telecomunicaciones. 
- Servicios de acceso a Internet.

- Sistemas de Información.

- Recursos humanos especializados en todas las áreas de las Tecnologias de la Información.

- Fuerza de trabajo capaz de asimilar las tecnologias de información y de producir con calidad.

La conectividad a redes mundiales de información abarca el hardware, software y el trabajo técnico necesario para la instalación, operación y mantenimiento de una infraestructura de redes informáticas y de sus consecuentes servicios, contemplando los aspectos legales, comerciales y éticos correspondientes.

La formación de recursos humanos que demandará la Sociedad de la Información, será la más importante de la que pudo requerir cualquier otra época de la historia de la humanidad. Cientificos, ingenieros, educadores, amas de casa, jubilados, nifios y todo el conjunto de la población, demandarán y necesitarán formación en aspectos del uso de las nuevas tecnologias de la información. Por otro lado, los profesionales y técnicos del mencionado sector, requerirán de una permanente actualización de sus conocimientos, para que sean capaces de producir con calidad y competitividad.

En aquellos paises en los cuales las nuevas infraestructuras de información están siendo implantadas y donde más han avanzado las concepciones de la Sociedad de la Información, el papel delos gobiemos ha sido fundamental, tanto en lo concerniente a la formulación e implantación de politicas nacionales de información, como también en su carácter de gran usuario de estas tecnologías. Las fuerzas del mercado por sí mismas no necesariamente promueven las mejores soluciones para el conjunto de la sociedad, es más, los requisitos tecnológicos de las infraestructuras de información para las actividades culturales, de educación y de investigación-desarrollo, son mucho más exigentes que aquellas que soportan actividades comerciales clásicas, que son satisfechas por el propio mercado. Por lo que se debe insistir, en el papel promotor y regulador que deben jugar los gobiemos. Los gobiemos pueden y deben tomar la iniciativa e incrementar la utilización de las nuevas tecnologias de la información para aumentar su propia eficiencia, para promover una mayor efectividad en sus acciones, para realizar proyectos de desarrollo de gran impacto social y para impulsar el sistema educacional a todos los niveles, aspecto fundamental para el establecimiento de una sociedad basada en el conocimiento. 


\section{BIBLIOGRAFÍA REFERENCIAL}

AGUDO, Alvaro. Ética en la Sociedad de Información. Reflexiones desde América Latina y El Caribe. En Boletin INFOLAC, Vol. 14, N4, 2001.

BAGGIO, Rodrigo. Las nuevas tecnologías y el tercer mundo: ¿una oportunidad? Nueva Revista de Politica, Cultura y Arte, N ${ }^{\circ} 70$, p. 137139, jul.-ago. 2000.

CARIDAD SEBASTIÁN, Mercedes (coord.) La Sociedad de la Información: política, tecnologia e industria de los contenidos. Madrid: Fundación Ramón Areces, 1999.

CASTELS, Manuel. La era de la información. Madrid : Alianza Editorial, 1998.

CRUZ Rosalba; ZETTER, Julio. Las Políticas de Información de la UNESCO y su repercusiónen América Latina. En Esp. Doc. Cient., UNAM, México, abril 1992.

CUBILLO, Julio. Estrategias para crear Sistemas de Información y Conocimientos en los 90, algunas reflexiones. En Ciencias de la Información. Vol. 26. N 4, dic. 1995.

FERNÁNDEZ-ABALdígdsidrechasóciedad/deda información en América Latina y el Caribe. Estrategia regional 2000-2001. En: Boletin INFOLAC, Vol. 12, №3, 1999.

MONTVILOFF, Victor. Politicas nacionales de información. Manual para la formulación, aprobación, ejecución y funcionamiento de una politica nacional de información. PGI y UNISIST. París: UNESCO, 1990.

NAISHBITT, John. Megatendencias 2000. México: Ed,. Norma, 1998.

NEGROPONTE, Nicholas. El mundo digital. Barcelona: Ediciones B, Grupo Zeta, 1995.

OLIVÉ GARCÍA, Aleida. La información en el desarrollo nacional: Desafio y 
alternativa para las economías emergentes. En Boletín INFOLAC, Vol. 12, $\mathrm{N}^{\circ} 1,1999$.

Reunión de Expertos para la definición de Estrategias Regionales de Información en América Latina y el Caribe, Caracas, marzo 2-4, 1993. Informe final PGI/LAC. Caracas, 1993.

RODRIGUEZ REYES, Victórico. Los servicios de información en el próximo milenio. En Boletín INFOLAC. Vol. 10, No 4, 1997. pp. 4-12.

SAKAIYA, Taichi. Historia del futuro, la sociedad del futuro. Santiago de Chile: Editorial Andrés Bello, 1995.

TOFLER, Alvin. La tercera ola. Barcelona: Ed. Guadarrama, 1990.

YONEJI, Mazuda. La sociedad informatizada como sociedad post-industrial. Barcelona: Editorial Fundesco, 1984. 